\title{
High-Flow Nasal Oxygen Therapy: One More Chance for Extubation?
}

Apart from the clinical conditions that require immediate ventilator support, conventional oxygen therapy (COT) via a face mask or nasal cannula is considered the "firststep approach" in the escalating therapy for the management of acute respiratory failure (ARF) to buy time for the etiologic therapy to reverse the triggering cause of the acute decompensation. ${ }^{1}$ Once physicians realize that only COT is not enough to properly and quickly correct the impaired lung gas exchange and to reduce the burden of respiratory distress, noninvasive ventilation (NIV) becomes the next option, the aim of which is to avoid the need for invasive mechanical ventilation and to prevent its life-threatening complications. ${ }^{2}$ Conversely, when an intubated and invasively ventilated patient is ready to be extubated, the transition from being assisted mechanically to spontaneous breathing on COT may be facilitated by the application of NIV, especially in some categories of subjects with underlying chronic cardiopulmonary diseases. ${ }^{3,4}$ Outside the do-notintubate setting, the failure of noninvasive strategies (COT and NIV) in escalating and de-escalating pathways leads to mandatory invasive mechanical ventilation via endotracheal intubation and re-intubation, respectively. ${ }^{1,5}$

Even though in the last 2 decades NIV has dramatically changed the epidemiology of mechanical ventilation in an expanding number of acute clinical scenarios, the chance of success with this ventilatory technique is variable and strongly dependent on several variables, such as the team's experience, patient-ventilator synchrony, air leaks, adequate equipment and environment, pathophysiology pattern, and timing and severity of ARF., ${ }^{2,6}$ Above all, the adherence to the scheduled ventilatory treatment is the crucial ingredient for successfully adapting to, maintaining, and weaning from a ventilator for the patient treated with NIV. $2,7,8$ As a matter of a fact, when NIV is or becomes a unviable therapeutic option for intolerant candidates, physicians may choose to keep COT for both patients who are still in the early stages of ARF when invasive mechanical ventilation is not still mandatory and those

The author has disclosed no conflicts of interest.

Correspondence: Raffaele Scala MD, Via Lorenzetti, 9, 52100 Arezzo, Italy. E-mail: raffaele_scala@hotmail.com.

DOI: $10.4187 /$ respcare. 03157 patients who have been successfully weaned from invasive mechanical ventilation without signs of postextubation respiratory distress.

Unfortunately, COT has several drawbacks: (1) limited amount of oxygen supplied with a maximum flow of $15 \mathrm{~L} /$ min; (2) imprecision and instability of the delivered $\mathrm{F}_{\mathrm{IO}_{2}}$ depending on the variability of the patient's breathing pattern; (3) risk of $\mathrm{CO}_{2}$ rebreathing with reservoir devices; (4) poor patient mask tolerance, especially for long-term treatment, and interference with eating, drinking, and speaking; (5) insufficient heating and humidification of the administered dry gas; and (6) substantial mismatch between the oxygen flow and the patient's inspiratory demand. Concerning the latter, given the fact that the patient's peak inspiratory demand may vary between 30 and $120 \mathrm{~L} / \mathrm{min}$ during an attack of ARF, only a small amount of the inspired gas (ie, $<10 \%$ ) can be properly humidified and oxygenated. ${ }^{1}$ This may result in severe mucosal damage and impairment of the physiological mechanisms of mucociliary clearance. ${ }^{9}$ Finally, COT is unable to unload the huge work of respiratory muscles ${ }^{10}$ and may contribute to an increase in $\mathrm{P}_{\mathrm{aCO}_{2}}$ level with a contextual drop in $\mathrm{pH}$ and the risk of precipitating the need for mechanical ventilation particularly in patients with acute-on-chronic hypercapnic respiratory failure. ${ }^{1,11}$

Very recently, an alternative system to deliver oxygen therapy has received increased attention in clinical practice due to its technical properties of potentially overcoming the intrinsic limitations of COT devices for the treatment of severely hypoxemic ARF patients who no longer require immediate ventilator support. The high-flow nasal oxygen cannula (HFNC) is a new technological system that can deliver up to $100 \%$ heated and humidified oxygen at a maximum gas flow of $60 \mathrm{~L} / \mathrm{min}$ via nasal prong or cannula; moreover, it is provided with an air-oxygen blender that allows the administration of gas with a preset $\mathrm{F}_{\mathrm{IO}_{2}}$ ranging from 0.21 to $1 .{ }^{12-15}$

Compared to COT, HFNC has several theoretical physiological advantages: (1) chance of delivering higher levels of $\mathrm{F}_{\mathrm{IO}_{2}}$ with good reliability; (2) washing out of the pharyngeal dead space with improved $\mathrm{CO}_{2}$ clearance ${ }^{12,13}$; (3) efficient humidification and heating of the delivered air-oxygen mixture with prevention of dryness-induced mucosal and mucociliary damage and facilitation of secretion 


\section{High-Flow Nasal Oxygen Therapy}

removal ${ }^{16}$; (4) good patient acceptance without interference with eating, drinking, and speaking; (5) possibility of matching the HFNC flow to the patient's inspiratory demand and/or degree of respiratory distress; and (6) generation of a flow-dependent PEEP (up to a median of $7.4 \mathrm{~cm} \mathrm{H}_{2} \mathrm{O}$ at $60 \mathrm{~L} / \mathrm{min}$ ) with a contextual upper airway resistance reduction (stenting effect) and alveolar recruitment (PEEP effect). ${ }^{17-19}$ Due to its favorable actions on respiratory work, HFNC appears to provide an increased level of respiratory support in comparison with COT devices and has been proposed as a potential alternative respiratory support to NIV, especially in pediatric patients when mechanical ventilation is not mandatory both to prevent endotracheal intubation and to facilitate weaning from invasive mechanical ventilation. Furthermore, the possibility of maintaining high-flow oxygen supplementation in patients who are going to be intubated or re-intubated is another point in favor of HFNC in terms of the safety management of critically hypoxemic patients. ${ }^{14,15}$

The amount of clinical data (even if mostly uncontrolled) is increasing on the feasibility, efficacy, and tolerance of HFNC in healthy volunteers ${ }^{17}$; in large groups of pediatric ARF subjects ${ }^{20,21}$; in hypoxemic ARF of different etiology with the aim of reducing the escalating ventilatory therapy (ie, NIV and invasive mechanical ventilation) ${ }^{22-24}$; in donot-intubate patients as an alternative to NIV ${ }^{25}$; in endstage chronic cardiopulmonary diseases with ARF 26,27 ; in post-cardiac surgery patients as prophylactic support to reduce the need of mechanical ventilation ${ }^{28}$; and during bronchoscopy in high-risk ARF patients. ${ }^{29}$

Experience with the use of HFNC after a successful spontaneous breathing trial to prevent extubation failure in adults is lacking. This is a crucial issue in respiratory intensive care medicine. In fact, ARF after a planned extubation is reported to be a common event, leading to re-intubation, and can occur in as many as 3-20\% of extubated patients. ${ }^{30}$ These re-intubated patients have higher morbidity, mortality, and hospitalization charges and increased hospital stay. ${ }^{31}$ NIV has been shown to effectively work as a prophylactic tool to prevent extubation failure in selected high-risk subject categories. ${ }^{2-4}$ However, some patients could not benefit from a trial of NIV after successful weaning because of their very poor tolerance, and the role of NIV in preventing re-intubation in de novo hypoxemic patients with ARF is still unclear. ${ }^{6}$ This niche of patients is likely to benefit from the early prophylactic use of HFNC after extubation given the highlighted physiologic features provided by this new oxygen device. Accordingly, at least in some specific scenarios, HFNC may be seen as an additional step after COT with the goal of preventing mechanical ventilation in escalating and deescalating support strategies (Fig. 1).

In this issue of Respiratory CARE, Rittayamai et al ${ }^{32}$ report on a short-term randomized crossover physiologic

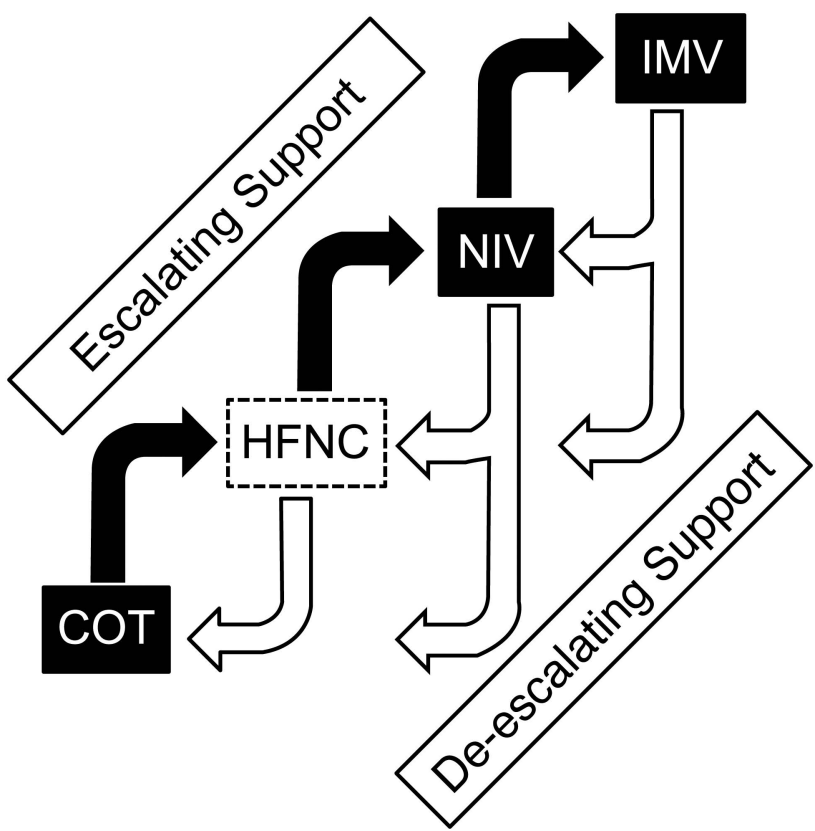

Fig. 1. Escalating (black arrows) and de-escalating (white arrows) support in acute respiratory failure. COT = conventional oxygen therapy; HFNC = high-flow nasal oxygen cannula; NIV = noninvasive ventilation; IMV = invasive mechanical ventilation.

trial in their respiratory ICU with the aim of comparing the efficacy of HFNC and COT in terms of clinical, physiologic, and subjective comfort data in a group of 17 consecutive heterogeneous patients $(35.2 \%$ with COPD exacerbation) who had undergone a successful spontaneous breathing trial. Subjects with hemodynamic instability, a decreased level of consciousness, and tracheostomy and those who failed to cooperate or were pregnant were excluded. After endotracheal extubation, the recruited patients were randomized to Protocol A $(n=9)$ or Protocol B $(n=8)$. In Protocol A, oxygen therapy was delivered via $\mathrm{HFNC}$ at an initial flow of $35 \mathrm{~L} / \mathrm{min}$ with $\mathrm{F}_{\mathrm{IO}_{2}}$ able to achieve $\mathrm{S}_{\mathrm{pO}_{2}} \geq 94 \%$ within the first $5 \mathrm{~min}$, maintaining this setting for $30 \mathrm{~min}$, and then COT was administered via a non-rebreathing face mask at a flow of 6-10 L/min to achieve $\mathrm{S}_{\mathrm{pO}_{2}} \geq 94 \%$ for $30 \mathrm{~min}$. In Protocol $\mathrm{B}$, oxygen was delivered via COT and then HFNC following the same setting and scheduled time.

Compared with COT, at the end of the trial, the use of HFNC was associated with a significant reduction in dyspnea $(\mathrm{P}=.04)$, breathing frequency $(\mathrm{P}=.009)$, and heart rate $(\mathrm{P}=.006)$, with a nonsignificant trend toward a better subjective comfort $(\mathrm{P}=.07)$. Interestingly, these advantages occurred very early in the time course of the study, after only $5 \mathrm{~min}$ for breathing frequency and after $10 \mathrm{~min}$ for heart rate and dyspnea. No significant differences emerged between the two groups with regard to oxygen saturation and blood pressure. No major adverse effects were reported with HFNC. Only 2 subjects complained 


\section{High-Flow Nasal Oxygen Therapy}

that the gas flow was too high and the temperature too warm, but both tolerated HFNC until the end of the scheduled treatment. No patient required escalating therapy (NIV or re-intubation) for extubation failure. According to their encouraging findings, the authors suggested that HFNC may have a role after endotracheal extubation; however, they call for large randomized control studies to better define the benefits of this device in this population.

The paper by Rittayamai et $\mathrm{al}^{32}$ may be seen as one of the first bricks in the wall of a still unexplored new potential application field of HFNC in adults. So far, literature demonstrating the role of HFNC in postextubation time is scare. In the only previous randomized controlled published study, showing a similar design, Tiruvoipati et al $^{33}$ did not report any significant differences in gas exchange, breathing frequency, or hemodynamics between HFNC and COT delivered to a larger group of 50 extubated patients recruited after a stabilization period of $30 \mathrm{~min}$. There was a significant difference $(\mathrm{P}=.01)$ only in tolerance and a trend $(\mathrm{P}=.09)$ toward better comfort with HFNC. In contrast to Rittayamai et al, Tiruvoipati et al did not include COPD patients and used higher flows to administer COT (30 vs $8 \mathrm{~L} / \mathrm{min}$ ).

Unfortunately, the study by Rittayamai et $\mathrm{al}^{32}$ has important caveats that strongly reduce the strength of the take-home message of its findings. First, if one understands correctly that the authors could not design a blinded study with two different technical devices to administer oxygen, the lack of a washout period between the two interventions may have resulted in an underestimation of the differences between the treatments because of the carryover effect.

Second, the application of such different flows may have largely favored HFNC over the COT low-flow device (36.8 vs $8 \mathrm{~L} / \mathrm{min})$. A protocol comparing the two devices with increasing oxygen flows (eg, 15, 20, $30 \mathrm{~L} /$ min) would have avoided this bias and, at the same time, would have given further information about the potential flow-dependent benefits of HFNC over COT (ie, PEEP effect and $\mathrm{CO}_{2}$ washout with the latter).

Third, the study did not consider the impact that the open-mouth situation has on the physiologic effects of HFNC (eg, reduced levels of PEEP). On the other hand, it has been demonstrated that $\mathrm{F}_{\mathrm{IO}_{2}}$ delivered by COT via face mask does not change with closed compared to opened mouth. ${ }^{34}$ Mouth-breathing patients are likely to benefit less from HFNC application after extubation.

Fourth, the comparability of the two groups of patients may be questionable given the fact that several parameters at enrollment were not available: arterial blood gases, body mass index, acute severity score, sensorium level, eventual use of sedation and analgesia, motor autonomy, and previous use of domiciliary oxygen and/or mechanical ventilation, with the lack of data on arterial blood gases being the most important. $\mathrm{P}_{\mathrm{aO}_{2}}$ level and $\mathrm{P}_{\mathrm{aO}_{2}} / \mathrm{F}_{\mathrm{IO}}$, the best physiologic indexes to assess lung oxygenation capability, were not available, and the oxygenation effects of HFNC and COT were estimated only with $\mathrm{S}_{\mathrm{pO}_{2}}$, the reliability of which may be impaired in several common conditions (eg, poor peripheral perfusion, shift of hemoglobin-oxygen saturation curve, hyperbilirubinemia, artifacts). Moreover, the effective $\mathrm{F}_{\mathrm{IO}_{2}}$ delivered by both oxygen devices was not measured, and the lack of these data introduces another bias in the comparability of the two groups. Differences in $\mathrm{P}_{\mathrm{aCO}_{2}}$ and $\mathrm{pH}$ behavior with HFNC versus COT may have given critical information on the potential $\mathrm{CO}_{2}$-rebound effects that high-flow oxygen therapy is likely to have, especially in hypercapnic patients with COPD exacerbation. Unfortunately, the prevalence rate of hypercapnic patients in the study population is unknown.

Fifth, due to the relatively low values of dyspnea (Borg scale $<3$ points), breathing frequency ( $<25$ breaths $/ \mathrm{min}$ ), and heart rate $(<100$ beats/min), one could reasonably argue that the patients treated in the study were not in respiratory distress, and presumably similar results could have been achieved with COT. Accordingly, no patient experienced extubation failure and required escalating therapy (NIV or re-intubation). In this context, it is difficult to assess the clinical importance of the small, even if statistically significant, improvement in dyspnea (1 point according to the Borg scale), breathing frequency (4 breaths/ min), and heart rate (6 beats/min) obtained with HFNC over COT.

Finally, the potential benefit of HFNC over COT in facilitating the removal of secretions due to greater humidification and heating of the inhaled gases has not been explored. This could be a crucial point for successful extubation in COPD patients with tenacious viscous mucus and reduced cough efficiency.

Important questions remain concerning the possible role of HFNC in postextubation time that the study by Rittayamai et $\mathrm{al}^{32}$ left unresolved. Does a subset of patients exist who are likely to obtain a greater benefit from HFNC (eg, those with non-hypercapnic/hypoxemic ARF, hypersecretion, or high level of dyspnea)? Is it reasonable to hypothesize the use HFNC as an alternative to NIV in maskintolerant patients, especially with a milder degree of postextubation distress? Is there a risk that a prolonged HFNC trial could unduly delay escalating therapy with a worse prognosis? Do physicians have reliable predictors of HFNC failure that should be followed to choose the right timing to escalate to mechanical ventilation? What are the overall costs (instrumental and human) for HFNC compared to those required for COT and NIV?

It is still unclear whether HFNC should be considered as one more chance for achieving a greater success rate in postextubation strategy. Thanks to the study by Rittayamai 


\section{High-Flow Nasal Oxygen Therapy}

et al, ${ }^{32}$ a new research field for the application of HFNC in extubated adults has just begun!

Raffaele Scala MD

Pulmonary Division and Respiratory Intensive Care Unit San Donato Hospital Arezzo, Italy

\section{REFERENCES}

1. O'Driscoll BR, Howard LS, Davison AG. BTS guideline for emergency oxygen use in adult patients. Thorax 2008;63(Suppl 6):vi1vi68.

2. Hess DR. Noninvasive ventilation for acute respiratory failure. Respir Care 2013;58(6):950-972.

3. Glossop AJ, Shephard N, Bryden DC, Mills GH. Non-invasive ventilation for weaning, avoiding reintubation after extubation and in the postoperative period: a meta-analysis. Br J Anaesth 2012;109(3): 305-314.

4. Agarwal R, Aggarwal AN, Gupta D, Jindal SK. Role of noninvasive positive-pressure ventilation in postextubation respiratory failure: a meta-analysis. Respir Care 2007;52(11):1472-1479.

5. Curtis JR, Cook DJ, Sinuff T, White DB, Hill N, Keenan SP, et al. Noninvasive positive pressure ventilation in critical and palliative care settings: understanding the goals of therapy. Crit Care Med 2007;35(3):932-939.

6. Nava S, Hill N. Non-invasive ventilation in acute respiratory failure. Lancet 2009;374(9685):250-259.

7. Carlucci A, Richard JC, Wysocki M, Lepage E, Brochard L, SRLF Collaborative Group on Mechanical Ventilation. Noninvasive versus conventional mechanical ventilation. An epidemiologic survey. Am J Respir Crit Care Med 2001;163(4):874-880.

8. Scala R, Coniglio G, Naldi M, Elliott MW. Compliance during noninvasive positive pressure ventilation (NIPPV) in acute hypercapnic respiratory failure (ARF). Eur Respir J 2004;24(Suppl 48):475s$476 \mathrm{~s}$.

9. Esquinas Rodriguez AM, Scala R, Soroksky A, BaHammam A, de Klerk A, Valipour A, et al. Clinical review: humidifiers during noninvasive ventilation-key topics and practical implications. Crit Care 2012;16(1):203

10. L'Her E, Deye N, Lellouche F, Taille S, Demoule A, Fraticelli A, et al. Physiologic effects of noninvasive ventilation during acute lung injury. Am J Respir Crit Care Med 2005;172(9):1112-1118.

11. Austin MA, Wills KE, Blizzard L, Walters EH, Wood-Baker R. Effect of high flow oxygen on mortality in chronic obstructive pulmonary disease patients in prehospital setting: randomised controlled trial. BMJ 2010;341:c5462.

12. Kernick J, Magarey J. What is the evidence for the use of high flow nasal cannula oxygen in adult patients admitted to critical care units? A systematic review. Aust Crit Care 2010;23(2):53-70.

13. Lee JH, Rehder KJ, Williford L, Cheifetz IM, Turner DA. Use of high flow nasal cannula in critically ill infants, children, and adults: a critical review of the literature. Intensive Care Med 2013;39(2): 247-257.

14. Ward JJ. High-flow oxygen administration by nasal cannula for adult and perinatal patients. Respir Care 2013;58(1):98-122.

15. Gotera C, Díaz Lobato S, Pinto T, Winck JC. Clinical evidence on high flow oxygen therapy and active humidification in adults. Rev Port Pneumol 2013;19(5):217-227.

16. Sztrymf B, Messika J, Bertrand F, Hurel D, Leon R, Dreyfuss D, Ricard JD. Beneficial effects of humidified high flow nasal oxygen in critical care patients: a prospective pilot study. Intensive Care Med 2011;37(11):1780-1786.

17. Groves N, Tobin A. High flow nasal oxygen generates positive airway pressure in adult volunteers. Aust Crit Care 2007;20(4):126131.

18. Parke R, McGuinness S, Eccleston M. Nasal high-flow therapy delivers low level positive airway pressure. Br J Anaesth 2009;103(6): 886-890.

19. Corley A, Caruana LR, Barnett AG, Tronstad O, Fraser JF. Oxygen delivery through high-flow nasal cannulae increases end-expiratory lung volume and reduces respiratory rate in post-cardiac surgical patients. Br J Anaesth 2011;107(6):998-1004.

20. Schibler A, Pham TM, Dunster KR, Foster K, Barlow A, Gibbons K, Hough JL. Reduced intubation rates for infants after introduction of high-flow nasal prong oxygen delivery. Intensive Care Med 2011; 37(5):847-852.

21. Manley BJ, Owen LS, Doyle LW, Andersen CC, Cartwright DW, Pritchard MA, et al. High-flow nasal cannulae in very preterm infants after extubation. N Engl J Med 2013;369(15):1425-1433.

22. Roca O, Riera J, Torres F, Masclans JR. High-flow oxygen therapy in acute respiratory failure. Respir Care 2010;55(4):408-413.

23. Sztrymf B, Messika J, Bertrand F, Hurel D, Leon R, Dreyfuss D, Ricard JD. Beneficial effects of humidified high flow nasal oxygen in critical care patients: a prospective pilot study. Intensive Care Med 2011;37(11):1780-1786.

24. Rello J, Pérez M, Roca O, Poulakou G, Souto J, Laborda C, et al. High-flow nasal therapy in adults with severe acute respiratory infection: a cohort study in patients with 2009 influenza A/H1N1v. J Crit Care 2012;27(5):434-439.

25. Peters SG, Holets SR, Gay PC. High-flow nasal cannula therapy in do-not-intubate patients with hypoxemic respiratory distress. Respir Care 2013;58(4):597-600.

26. Boyer A, Vargas F, Delacre M, Saint-Léger M, Clouzeau B, Hilbert G, Gruson D. Prognostic impact of high-flow nasal cannula oxygen supply in an ICU patient with pulmonary fibrosis complicated by acute respiratory failure. Intensive Care Med 2011;37(3):558-559.

27. Roca O, Pérez-Terán P, Masclans JR, Pérez L, Galve E, Evangelista A, Rello J. Patients with New York Heart Association class III heart failure may benefit with high flow nasal cannula supportive therapy: high flow nasal cannula in heart failure. J Crit Care 2013;28(5):741746.

28. Parke R, McGuinness S, Dixon R, Jull A. Open-label, phase II study of routine high-flow nasal oxygen therapy in cardiac surgical patients. Br J Anaesth 2013;111(6):925-931.

29. Lucangelo U, Vassallo FG, Marras E, Ferluga M, Beziza E, Comuzzi $\mathrm{L}$, et al. High-flow nasal interface improves oxygenation in patients undergoing bronchoscopy. Crit Care Res Pract 2012;2012:506382. doi:10.1155/2012/506382.

30. Epstein SK. Extubation. Respir Care 2002;47(4):483-492; discussion 493-495.

31. Rothaar RC, Epstein SK. Extubation failure: magnitude of the problem, impact on outcomes, and prevention. Curr Opin Crit Care 2003; 9(1):59-66.

32. Rittayamai N, Tscheikuna J, Rujiwit P. High-flow nasal oxygen cannula versus conventional oxygen therapy after endotracheal extubation: a randomized crossover physiologic study. Respir Care 2014;59(4):485-490.

33. Tiruvoipati R, Lewis D, Haji K, Botha J. High-flow nasal oxygen vs high-flow face mask: a randomized crossover trial in extubated patients. J Crit Care 2010;25(3):463-468.

34. Chanques G, Riboulet F, Molinari N, Carr J, Jung B, Prades A, et al. Comparison of three high flow oxygen therapy delivery devices: a clinical physiological cross-over study. Minerva Anestesiol 2013; 79(12):1344-1355. 